\title{
Assessing the effectiveness of a real-time control method for Marina Reservoir management
}

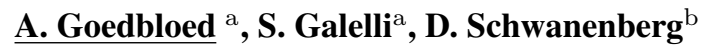 \\ ${ }^{a}$ Singapore-Delft Water Alliance, National University of Singapore, Singapore \\ ${ }^{b}$ Operational Water Management, Deltares, Delft, The Netherlands \\ Email: a.goedbloed@nus.edu.sg
}

\begin{abstract}
Marina Reservoir is one of the largest fresh water body in Singapore, recently constructed with the purpose of increasing the drinking water supply and Singapore's self-sufficiency. Besides this strategic role, the reservoir also serves for floods control and lifestyle attraction. The largest portion of the inflow volumes comes from five main uncontrolled catchments, which show fast-varying discharge variations due to the strong storms affecting this region. Moreover, highly paved areas cause rapid runoff processes, with a concentration time lower than one hour. Such hydrological context creates difficulties in satisfying the operational objectives and provides a challenging environment for the application of real-time control methods.

With the purpose of evaluating the potential of real-time control for the optimal operation of Marina Reservoir, this work adopts Model Predictive Control (MPC), a form of deterministic control that employs the current state of the system, the future inflows trajectory furnished by a predictive model and a further model describing the internal dynamics of the controlled sub-system to determine an optimal control sequence over a finite prediction horizon. The rationale behind the choice of MPC is that this method is characterized by reduced computational requests and the capability of exploiting any exogenous information, as precipitation or evaporation measurements, that allow for an accurate prediction of the inflow events, which is crucial for an effective management of fast-varying hydrological systems.

In the present application, Marina Reservoir catchment is described with a data-driven rainfall-runoff model (in the form of M5 model trees) that fully exploits the hydro-meteorological information available in real-time, thus enhancing the accuracy of the inflow prediction. The model is combined with a non-linear MPC scheme that optimizes the barrage operation according to the different operational objectives. Preliminary results show the effectiveness of the proposed control method, which outperforms the currently-used operating rules.
\end{abstract}

Keywords: Multi-purpose reservoir operation, model predictive control, inflow predictions, multiobjective optimization 


\section{INTRODUCTION}

During the last decades Singapore water security has been largely dependent on water transfers from the neighbouring Malaysia'a state of Johor. To increase its self-sufficiency, Singapore has recently adopted the so-called Four National Taps Strategies, which detect local catchment areas, imported water, reclaimed and desalinated water as the four national 'taps' (for further details see Kristiana et al. (2011) and references therein). Among these, the maximization of water yields from local catchments is one of the most potentially important sources, and with the inclusion of Marina Reservoir the Singapore's effective catchment area is now increased to about $50 \%$ of the total available. Marina Reservoir, created in late 2008 with the construction of a 350 m-wide barrage across Marina Channel, represents one of the largest fresh water bodies in Singapore. Besides this strategic water supply role, the reservoir has two further functions: floods control and lifestyle attraction. Indeed, the Marina Bay area coincides with most of Singapore's central business district and it is the site around which an ambitious urban development project is taking place.

To ensure the required fresh water storage as well as a sufficiently-large volume to buffer flood events, the gates and pumps in the barrage are operated with the purpose of maintaining a stable water level. However, satisfying this operational objective is not straightforward, mainly because of the extremely short concentration time of the catchment that causes high peaks of discharge over a period of few hours (Janssen et al., 2007). In this hydrological context the efficient operation of Marina Reservoir calls for the adoption of real-time control methods, which, unlike traditional off-line methods (see Castelletti et al. (2008) for a review), can exploit the availability of hydro-meteorological informations (e.g. precipitation or evaporation measurements) and inflow predictions.

Among the different real-time control methods (Pianosi and Soncini-Sessa (2009), and references therein), this work aims at assessing the potential of Model Predictive Control (MPC) for the optimal operation of the barrage gates and pumps (see van Overloop et al. (2010) for the application of MPC to a similar system, where tides influence gates use and where MPC is aimed at energy and water level control). MPC is a form of deterministic control that has become an industrial standard in process engineering (Mayne et al., 2000) and that is finding water resources systems as a novel domain of application. In the present work, the rationale behind the selection of MPC stands in its contained computational demand and capability of dealing with explicit constraints and inflow predictions, which are key for adopting a relatively-short decision time-step imposed by the fast-varying dynamics of Marina Reservoir catchment. Numerical simulations show that MPC, combined with a data-driven rainfall-runoff model (in the form of M5 model trees), is effective in anticipating the inflow events, thus outperforming the currently-used operating rules in maintaining the desired water level and minimizing the energy request due to the pumps usage.

\section{PROBLEM AND SOLUTION STRATEGY}

Unlike off-line optimal control methods, which aim at designing a temporal (periodic) sequence of control laws, each providing the optimal release decision as a function of the state of the system at a given time instant, real-time control methods are based on the idea of solving the control problem on-line, by exploiting the receding horizon principle and a model describing the dynamics of the controlled subsystem. According to this principle, at each decision time-step $t$, a real-time control problem is formulated over the finite horizon $[t, t+h]$. For each time $\tau$ in the horizon $[t, t+h]$, a prediction of the disturbance (i.e. inflow realization) is provided by a dynamic predictor that exploits the hydro-meteorological information available in real-time. Once the problem is solved, only the first control $u_{t}$ for the first time-step $[t, t+1)$ is applied, and at time $t+1$ a new problem is formulated and solved over the horizon $[t+1, t+h+1]$ with a new prediction of the inflow realization. In particular, an MPC problem is formulated as a deterministic open-loop control problem of the following form (Mayne et al., 2000)

$$
\min _{u_{t}, \ldots, u_{t+h-1}}\left[\sum_{\tau=t}^{t+h-1} g_{\tau}\left(x_{\tau}, u_{\tau}, \varepsilon_{\tau+1}\right)+\bar{g}_{t+h}\left(x_{t+h}\right)\right]
$$

subject to

$$
x_{\tau+1}=f_{\tau}\left(x_{\tau}, u_{\tau}, \varepsilon_{\tau+1}\right)
$$




$$
\begin{aligned}
& 0 \leqslant u_{\tau} \leqslant u^{\max } \\
& x_{t} \text { given }
\end{aligned}
$$

with $\tau=t, \ldots, t+h-1$; where $x_{\tau}$ is the state of the system and $f_{\tau}(\cdot)$ the corresponding state transition function, $u_{\tau}$ the control variable (belonging to the finite set $\left[0, u^{\max }\right]$ ), $\varepsilon_{\tau+1}$ the disturbance in the interval $[\tau, \tau+1), g_{\tau}(\cdot)$ the step-cost expressing the cost associated to the state transition from $\tau$ to $\tau+1$, and $\bar{g}_{t+h}$ the penalty function associated to the final state $x_{t+h}$.

This problem, also known as Naive Feedback Control (Bertsekas, 1976), can be solved by means of (nonlinear) mathematical programming methods and its resolution yields to a sequence of control decisions $u_{t}, \ldots, u_{t+h-1}$ (see Bertsekas (2005) for a detailed analysis of the relation between MPC and the closedloop formulation of a real-time control problem). Next two sections provide an overview of the process models adopted to describe the dynamics of the controlled sub-system and and the inflow process, as well as the results obtained by adopting the proposed MPC scheme.

\section{MARINA RESERVOIR WATER SYSTEM}

\subsection{Overview}

Marina Reservoir (Singapore) was created in late 2008 with the construction of a barrage that closed from the sea the former homonymous marina, with the purpose of creating a freshwater impound. Five main tributaries discharge water into the reservoir coming from a catchment of approximately $100 \mathrm{~km}^{2}$, about $1 / 7^{\text {th }}$ of Singapore total area. The catchment, mainly consisting of urbanized land, is characterized by the presence of three further reservoirs, only managed for drinking water production. These reservoirs receive water from a relatively small forested catchment and they are operated in such a way that their discharge to Marina Reservoir is rare and insignificant. The catchment drainage system consists of concrete lined canals, which make the concentration time extremely short (about 1 hour). Rainfall tends to come in high intensity events and discharges occur in high peaks over short periods, typically a few hours. Base flow is low and the upstream canals are mostly dry. In the downstream parts of the drainage system the bottom level of the canals is often lower than the reservoir level, with stagnant water being present during draught periods.

The barrage can be operated by actuating gates and pumps: the former are used during low tide events, while the latter can discharge water during high tide events when the sea water level is higher than the reservoir level. The main objective is to keep the water level within the desired range $([-0.2,+0.3] \mathrm{m}$ above the sea level), thus maintaining a sufficient volume of available fresh water while avoiding the risk of flood events (the flooding threshold is $1.1 \mathrm{~m}$ ). The second objective is the minimization of energy costs due to pumps usage. In principle the reservoir is operated according to a fixed set of operating rules: these are fixed-class, feed-back control laws that can react to the changes in the reservoir level, but cannot anticipate the release decisions on the basis of the prediction of future events. The operating rules are mainly aimed at maintaining the reservoir level within the desired range, while they make little distinction between the usage of pumps and gates (for further details see Smits et al. (2007)).

\subsection{Process Models}

The reservoir. Since the real-time control problem requires to account only for water quantity objectives, the reservoir model has a single state variable, i.e. the storage $s_{t}\left[\mathrm{~m}^{3}\right]$, whose dynamics is regulated by the following mass balance equation

$$
s_{t+1}=s_{t}+a_{t+1}-\left(r_{t+1}^{g}+r_{t+1}^{p}\right)
$$

where $a_{t+1}$ is the net inflow volume $\left[\mathrm{m}^{3} /\right.$ hour] in the interval $[t, t+1$ ) (including direct precipitation on the reservoir, evaporation and infiltration), and $r_{t+1}^{g}$ and $r_{t+1}^{p}$ are the release [ $\mathrm{m}^{3}$ /hour] from gates and pumps respectively.

The control variables $u_{t}^{g}$ and $u_{t}^{p}$ are the release decisions $\left[\mathrm{m}^{3} /\right.$ hour] from gates and pumps, taken on a hourly basis. The modelling time-step is thus equal to one hour. Due to the presence of physical constraints, the actual releases $r_{t+1}^{g}$ and $r_{t+1}^{p}$ do not coincide with the controls $u_{t}^{g}$ and $u_{t}^{p}$, and the total 
release $r_{t+1}$ (equal to $r_{t+1}^{g}+r_{t+1}^{p}$ ) is computed by means of the following release function

$$
r_{t+1}= \begin{cases}v\left(s_{t}, u_{t}^{g}, u_{t}^{p}, a_{t+1}, h_{t}^{s}\right) & \text { if }\left(u_{t}^{g}+u_{t}^{p}\right)<v\left(s_{t}, u_{t}^{g}, u_{t}^{p}, a_{t+1}, h_{t}^{s}\right) \\ V\left(s_{t}, u_{t}^{g}, u_{t}^{p}, a_{t+1}, h_{t}^{s}\right) & \text { if }\left(u_{t}^{g}+u_{t}^{p}\right)>V\left(s_{t}, u_{t}^{g}, u_{t}^{p}, a_{t+1}, h_{t}^{s}\right) \\ u_{t}^{g}+u_{t}^{p} & \text { otherwise }\end{cases}
$$

where $h_{t}^{s}[\mathrm{~m}]$ is the seal level ${ }^{1}$ at time $t$, while $v(\cdot)$ and $V(\cdot)$ are the minimum and maximum release that can be produced in the time interval $[t, t+1)$, namely two non-linear functions describing the relation between the controls $u_{t}^{g}$ and $u_{t}^{p}$ and the release $r_{t+1}$ (Castelletti et al., 2008).

The catchment. The inflow process to Marina Reservoir is modelled by means of M5 model trees, originally developed by Quinlan (1992), that combine the traditional structure of a decision tree with the possibility of generating linear regression functions at the leaves. M5, which have found successful applications in different hydrological problems (Solomatine and Xue (2004), and references therein), can be seen as a piecewise linear model, generally characterized by good prediction performance and simple decision structures with few leaves and corresponding linear models.

In the present application M5 are used to predict the inflow process to Marina Reservoir over a prediction horizon of 6 hours. The adopted modelling time-step corresponds to one-hour, so the total number of models used at each decision time-step corresponds to six, with the former providing the inflow prediction $\hat{a}_{t+1}$ in the time interval $[t, t+1)$ and the latter providing the prediction $\hat{a}_{t+6}$ in the time interval $[t+$ $5, t+6)$. The most relevant inputs to the models, selected on the basis of a correlation analysis, are two autoregressive terms, i.e. the measured inflows $a_{t}$ and $a_{t-1}$, and three precipitation values ${ }^{2}[\mathrm{~mm} / \mathrm{h}]$, at the current hour and at the previous 2 hours, i.e. $p_{t}, p_{t-1}$ and $p_{t-2}$. The ensemble of six models sharing these inputs and providing the predictions $\hat{a}_{t+1}, \ldots, \hat{a}_{t+6}$ will be referred to as Measured Data Ensemble (MDE).

In order to evaluate the effectiveness of the MPC scheme under different prediction scenarios, two further ensembles are created by hypothetical assuming that one hour and three hours perfect rainfall predictions are available: these two ensembles, named 1-hour Rainfall Prediction Ensemble (1-RPE) and 3-hours Rainfall Prediction Ensemble (3-RPE), share the same autoregressive terms of the first ensemble, but make use of the precipitation predictions $p_{t+1}$ and $p_{t+3}, p_{t+2}, p_{t+1}$, thus providing an upper bound of the inflows model performance (see Table 1 for an exhaustive comparison).

Table 1. Coefficient of determination $\mathrm{R}^{2}$, evaluated in $k$-fold cross-validation (with $k=10$ ) over the period April 2009 - March 2010, for the three ensembles described within the text.

\begin{tabular}{|c|c|c|c|c|c|c|}
\hline & $1-\mathrm{h}$ & $2-\mathrm{h}$ & $3-\mathrm{h}$ & $4-\mathrm{h}$ & $5-\mathrm{h}$ & $6-\mathrm{h}$ \\
\cline { 2 - 7 } MDE & 0.7171 & 0.1801 & 0.0934 & 0.0596 & 0.0418 & 0.0429 \\
\cline { 2 - 7 } 1-RPE & 0.9173 & 0.7262 & 0.1896 & 0.0897 & 0.0459 & 0.0443 \\
\cline { 2 - 7 } 3-RPE & 0.9173 & 0.9159 & 0.9151 & 0.7274 & 0.1527 & 0.0799 \\
\hline
\end{tabular}

\subsection{Control objectives}

The water level and pumps operational objectives are accounted for by defining two step-costs functions $g_{t}^{h}(\cdot)$ and $g_{t}^{p}(\cdot)$ representing the costs associated with each state transition, namely

$$
g_{t}^{h}= \begin{cases}\left(h_{t}+0.2\right)^{2} & \text { if } h_{t}<-0.2 \\ \left(h_{t}-0.3\right)^{2} & \text { if } h_{t}>+0.3 \\ 0 & \text { otherwise }\end{cases}
$$

\footnotetext{
${ }^{1}$ The sea level variation is modelled in first approximation as a static astronomical tide signal.

${ }^{2}$ Rainfall data is available from several measurement locations throughout the catchment. These data are aggregated with Thiessen polygons.
} 
where $h_{t}$ is the reservoir level [m] (computed as the ratio between the storage $s_{t}$ and the surface $S$, equal to $2.5 \mathrm{~km}^{2}$ ); and

$$
g_{t}^{p}=\left(r_{t+1}^{p}\right)^{2}
$$

where $r_{t+1}^{p}$ is the actual release due to pumps operation.

The two step-costs are finally aggregated with the weighting method, i.e.

$$
g_{t}=\lambda \cdot g_{t}^{h}+(1-\lambda) \cdot g_{t}^{p}
$$

where $\lambda$ is a weight belonging to the interval $[0,1]$.

\section{RESULTS AND DISCUSSION}

Simulation analysis over the period April 2009 - March 2010 is used to comparatively evaluate the performance of the MPC scheme under alternative inflow prediction scenarios, evaluated with different combinations of weights used to aggregate the step-costs functions (see eq. (5)). For a further reference the performance (calculated via simulation) of the currently-used operating rules is also reported.

The base scenario that is applied is a perfect forecast of the inflow events. In Figure 1a the results of this scenario are shown: each line represents the performance of the controller under a specific prediction horizon (1, 2, 3, 6 or 12 hours ahead) with different weights applied to the control objectives. It can be seen that the MPC scheme outperforms the operating rules even with a 1 hour prediction horizon. With the same amount of pumped volume the water level objective can be improved, or by allowing the same cost on the water level objective the pumped volume can be significantly reduced. Moreover, it can also be noticed that by increasing the prediction horizon the performance improves as the controller can anticipate better on future inflow events; however a prediction horizon of more than 6 hours does not result in further improvements.

(a)

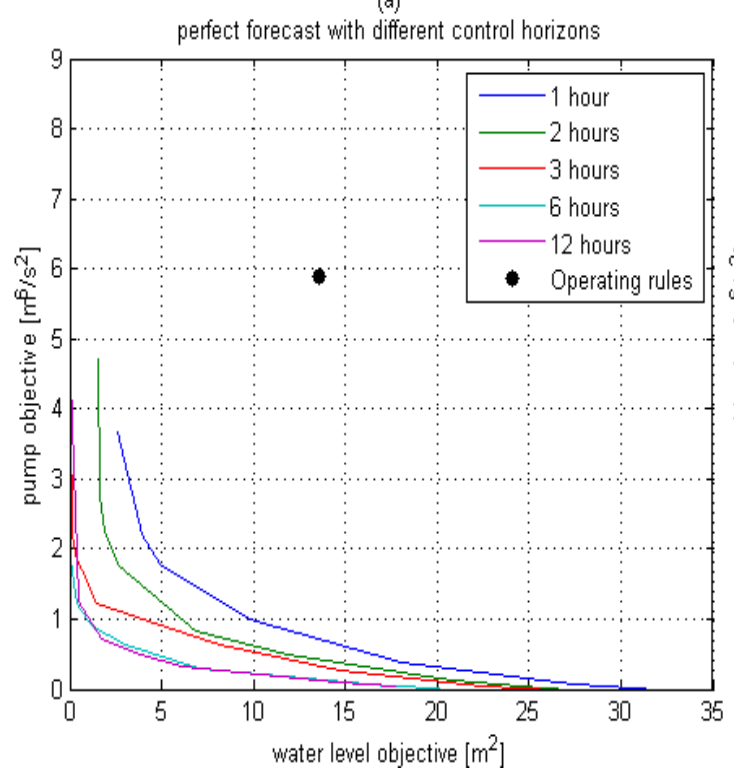

(b)

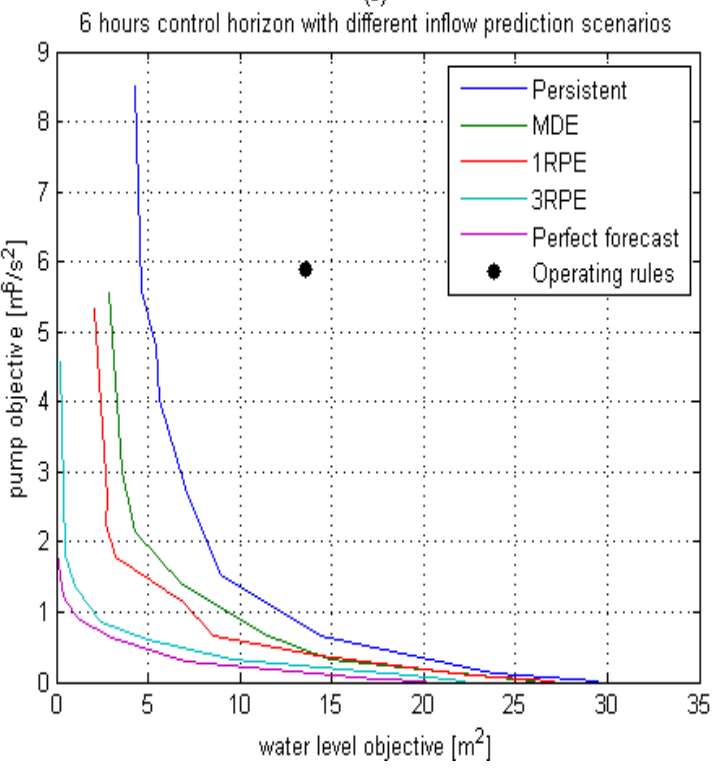

Figure 1. Pareto fronts obtained with a perfect inflow prediction and with different prediction horizons vs. the operating rules (a); Pareto fronts obtained with different inflow predictions scenarios and with a prediction horizon of 6 hours vs. the operating rules (b).

Figure $1 \mathrm{~b}$ shows the results of the different inflow prediction scenarios over a horizon of 6 hours. The 

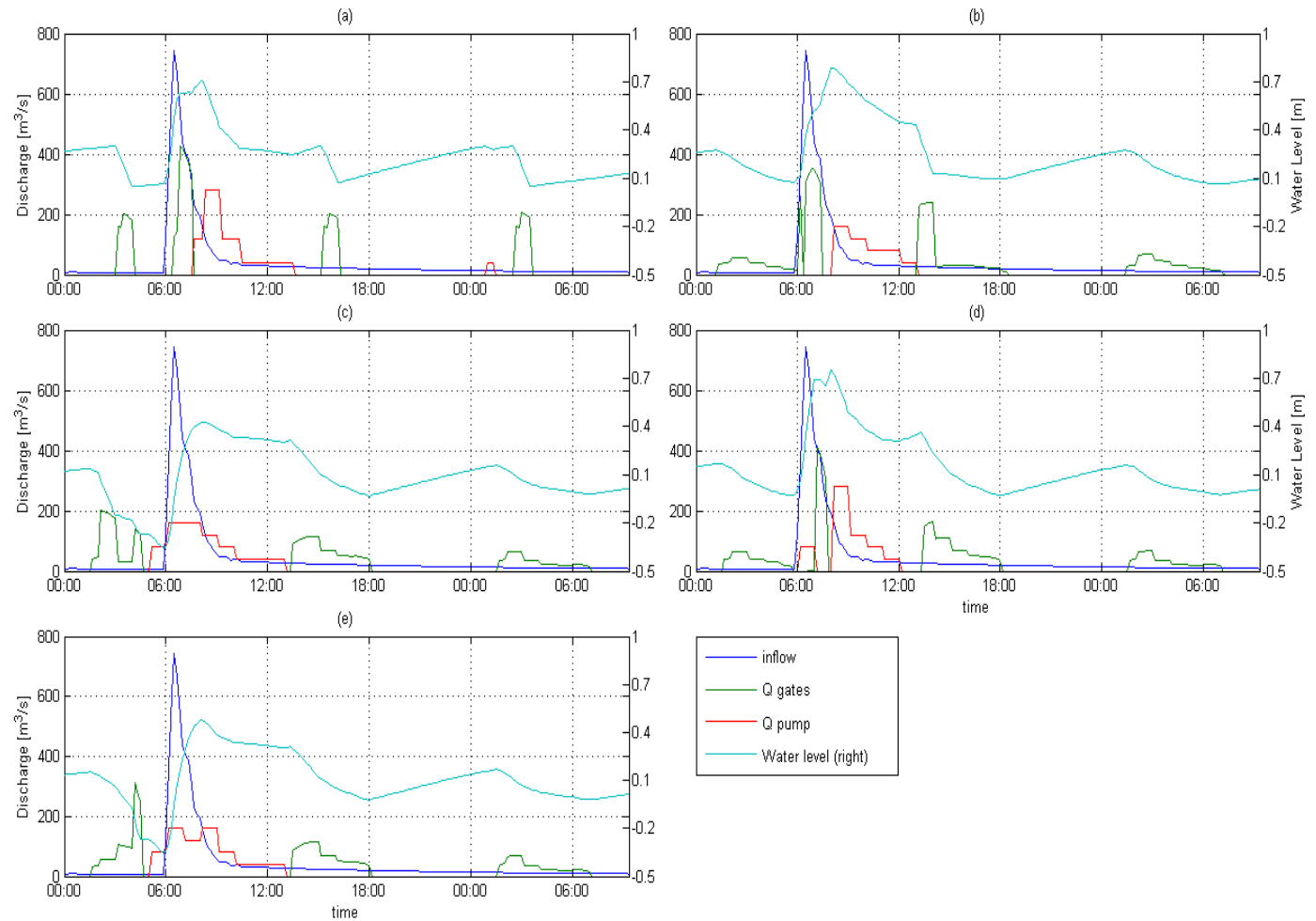

Figure 2. Sample of the control response with implementation of the operating rules (a), perfect forecast with a prediction horizon of 1 hour (b), perfect forecast with a prediction horizon of 6 hour (c), MDE scenario with a prediction horizon of 6 hours (d), 3-RPE scenario with a prediction horizon of 6 hours (e).

perfect forecast scenario marks the upper bound of the achievable performance, while a persistent prediction scenario, which extents the last measured inflow over the prediction horizon, serves as a lower bound of the performance. Again all different scenarios are superior when compared against the operating rules. The MDE scenario uses only measured data available at the moment of the optimization: as the concentration time of the system is about 1 hour, the inflow prediction is only accurate for the first 2 hours (see Table 1) and the MPC scheme, under this inflow scenario, does not largely improve the persistent scenario performance. On the other hand, the two scenarios that make use of a rainfall prediction show a significantly improvement in the control performance, and with the 3-RPE scenario the performance of the perfect forecast can almost be reached. These preliminary results demonstrate the potential of exploiting a rainfall prediction to improve operational performance. One remark that has to be made is that the analysis was done by assume a perfect rainfall forecast, and these results thus represent an upper bound in the performance that can be achieved.

Figure 2 shows a specimen of the trajectories of the control implementation under various scenarios. The operating rules (panel (a)) are characterized by low performance, since the controller does not anticipate the coming peak in the inflow and only starts acting when the water level already past the threshold. A similar behaviour is shown when adopting a perfect forecast of the inflow and a prediction horizon of only 1 hour (panel (b)). On the other hand, by enlarging the prediction horizon, better performance can be achieved. Figure $2 \mathrm{c}$ shows the control response with a perfect forecast of the inflow and a prediction horizon of 6 hours: it can be noticed that the controller lowers the water level in the reservoir to anticipate the inflow event, and the maximum water level is significantly lower than in the previous 2 samples. Figure $2 \mathrm{~d}$ shows the control response with the MDE scenario and a prediction horizon of 6 hours: despite adopting a longer horizon, the controller cannot anticipate the event, because the quality of the inflow 
prediction is not sufficient that far ahead in time. Figure 2e shows the control response with the 3-RPE scenario and a control horizon of 6 hours: in this case, by exploiting the rainfall prediction, the controller can anticipate the event and lower the water level in the reservoir beforehand so that a lower peak level is achieved.

\section{CONCLUSIONS}

This work represents a first attempt in applying a real-time control method for the operation of Marina Reservoir, Singapore. Results show that this approach can outperform the traditional operating rules and can be effective in satisfying the desired operational objectives, thanks to its capability in exploiting the inflow predictions provided by a data-driven rainfall-runoff model. In particular, the MPC control scheme permits to maintain the desired water level in the reservoir and to minimize the recourse to pumps usage during high-tide events. These results also show that it would be possible to increase the water level in the reservoir with the same flood protection but increased fresh water storage.

In order to account for the uncertainties associated to the inflow predictions, further research will be dedicated to the development of a stochastic control scheme and to the integration of rainfall predictions within the proposed control framework.

\section{ACKNOWLEDGEMENT}

The research presented in this work was carried out as part of the SDWA Multi-Objective MultipleReservoir Management research programme (R-303-001-005-272).

\section{REFERENCES}

Bertsekas, D. (1976). Dynamic Programming and Stochastic Control. New York, NY: Academic Press.

Bertsekas, D. (2005). Dynamic programming and suboptimal control: a survey from adp to mpc. European Journal of Control 11(4-5).

Castelletti, A., F. Pianosi, and R. Soncini-Sessa (2008). Water reservoir control under economics, social and environmental constraints. Automatica 44(6), 1595-1607.

Janssen, A., H. Ogink, G. Prinsen, and M. van der Vat (2007). Singapore marina reservoir study. hydrological and hydraulic model report. Research report Q4277, Deltares, Delft, The Netherlands. Available from authors.

Kristiana, R., J. Antenucci, and J. Imberger (2011). Sustainability assessment of the impact of the marina bay development on singapore: application of the index of sustainable functionality. International Journal of Environment and Sustainable Development 10(1), 1-35.

Mayne, D., J. Rawlings, C. Rao, and P. Scokaert (2000). Constrained model predictive control: stability and optimality. Automatica 36(6), 789-814.

Pianosi, F. and R. Soncini-Sessa (2009). Real-time management of a multi-purpose water reservoir with a heteroschedastic inflow model. Water Resources Research 45(W10430), -.

Quinlan, J. (1992). Learning with continuous classes. In Proceedings of the 5th Australian Joint Conference on Artificial Intelligence, November 16-18, Hobart, AUS, pp. 343-348.

Smits, J., J. van Beek, D. Twigt, and G. Prinsen (2007). Marina reservoir study. Research report Z4265.70, Deltares, Delft, The Netherlands. Available from authors.

Solomatine, D. and Y. Xue (2004). M5 model trees compared to neural networks: application to flood forecasting in the upper reach of the huai river in china. ASCE Journal of Hydrologic Engineering 9(6), 491-501.

van Overloop, P., R. Negenborn, S. Weijs, W. Malda, M. Bruggers, and B. D. Schutter (2010, September 8 -10). Linking water and energy objectives in lowland areas through the application of model predictive control. In 2010 IEEE International Conference on Control Applications (CCA), Yokohama, JP. 